\title{
The Significance for Natural Philosophy of the Move from Classical to Modern Physics
}

\author{
Grete Hermann ${ }^{1}$ \\ Published online: 10 December 2020 \\ (C) The Author(s) 2020
}

SUMMARY-This study shows how, despite the changes it has introduced, modern physics preserves certain fundamental ideas of classical physics (Bohr's correspondence principle). While it gives up much of the ideal of a mechanistic physics, it still remains tied to Kant's thesis that the forms of intuition and the categories are the necessary presuppositions for the knowledge of nature.

1. The development of modern physics has two distinctive aspects: on the one hand the demand for a revision of almost all fundamental assumptions on which the knowledge of nature has been based until now, and indeed for a revision based on experience; on the other hand the upholding of certain fundamental conceptions of classical physics, which finds its strongest expression in Bohr's correspondence principle. Modern physics presents us with the problem within natural philosophy of reconciling these two aspects.

2. The dualism between the wave and particle picture in quantum mechanics, with its consequences for [our] causal command of natural phenomena represents the strongest departure from the classical picture of nature. But this departure is closely connected to a series of earlier transformations in the picture of nature. The first step in this direction is taken in Maxwell's theory, which detaches the wave picture from the presupposition of a material support until then taken for granted. A further stage is the theory of relativity, with the demonstration that one cannot ascribe to matter a definite state of motion with respect to the 'ether'. Finally, while the steps up to now have brought the wave and particle picture more and more into opposition, quantum mechanics leads one to applying them again to one and the same atomic process.

Written by Grete Hermann, translated by Guido Bacciagaluppi, from the reprint in Herrmann, K. (Ed.) (2019). Grete Henry-Hermann: Philosophie-Mathematik-Quantenmechanik. Dordrecht: Springer, pp. 379-381. Originally published in German (with French summary) as 'Die naturphilosophische Bedeutung des Übergangs von der klassischen zur modernen Physik', Chapter XVII in Bayer, R. (Ed.) (1937). Travaux du IX Congrès International de Philosophie-Congrès Descartes. Vol. VII, Causalité et Déterminisme. Actualités Scientifiques et Industrielles, n. 536. Paris: Hermann et C ${ }^{\mathrm{ie}}$, pp. 99-101. Thanks to the editors for a careful reading of the translation.

Grete Hermann-deceased (1901-1984).

Grete Hermann

g.bacciagaluppi@uu.nl

1 Østrupgård, Denmark 
3. The starting point of this development is characterised by the abandonment of an old expectation, which in the Enlightenment dominated research in both physics and natural philosophy, namely the expectation that in the end physics would reduce completely to classical mechanics. What distinguishes this discipline is in fact its intuitive spatiotemporal modelling of natural phenomena. The construction of such a model proceeds by finding substances that fill space, determining the interactions obtaining between them, and the causal modifications of their state of motion thereby brought about. The fundamental concepts in the picture of nature of classical mechanics thus correspond so precisely to the Kantian forms of intuition and categories, that on the one hand Kant's philosophy has been seen as a justification for privileging classical mechanics, and on the other hand the discovery of the limits of classical mechanics has been taken as a refutation of Kant's insights.

4. If one analyses more closely the physical arguments that have led to this discovery, however, it turns out that Kant's fundamental concepts nowhere fail to apply. The experiments that ground the derivation of Maxwell's equations examine the interaction between material bodies; measurements of spatial and temporal relations in the theory of relativity presuppose the classical intuitions of Euclidean space and of an objective determination of simultaneity; quantum mechanics presupposes causal explanations in its theory of measurement, also for unpredictable events-indeed it is only by displaying these already known causes that it can establish the futility of a further search for causes, and thus the fundamental significance of the limits set to prediction.

5. In each of these disciplines however-in each case at a different place in the physical picture of nature-one relinquishes an assumption that is straightforwardly satisfied in classical mechanics and was tacitly the basis for the programme of reducing the whole of physics to classical mechanics. It is the assumption that every application of the classical connecting principles can be held on to unambiguously throughout the whole physical interpretation of natural phenomena. According to this assumption, what can be interpreted in some context as a substance, as simultaneous, as equally long, as matter in motion, must be interpreted in the same way in each observational context. It is in truth this assumption that has been abandoned beginning with Maxwell's theory and in ever more radical ways in modern physics. This is the explanation for the many natural-philosophical paradoxes that prevent the intuitive interpretation of the results of modern physics.

6. Modern physics thus indeed accords with Kant's thesis that the above-mentioned forms of intuition and categories are necessary preconditions for the knowledge of nature. Instead, the distinctive step that sets physics apart from the picture of nature of classical mechanics rules out any realist interpretation of physics that sees in the picture of nature provided by physics an adequate description of the phenomena. In this sense-when one examines its modes of argumentation closely-modern physics serves the purpose of extending and endorsing another Kantian idea: that of transcendental idealism-in the form given to it by the works of the Friesian school whereby knowledge of nature does not adequately capture reality, but in an imperfect way only extracts relational structures from it, the grounds of which remain undetermined within the framework of this knowledge.

Open Access This article is licensed under a Creative Commons Attribution 4.0 International License, which permits use, sharing, adaptation, distribution and reproduction in any medium or format, as long as you give appropriate credit to the original author(s) and the source, provide a link to the Creative Commons licence, and indicate if changes were made. The images or other third party material in this article are included in the article's Creative Commons licence, unless indicated otherwise in a credit line to the material. If material is not included in the article's Creative Commons licence and your intended use is not 
permitted by statutory regulation or exceeds the permitted use, you will need to obtain permission directly from the copyright holder. To view a copy of this licence, visit http://creativecommons.org/licenses/by/4.0/.

Publisher's Note Springer Nature remains neutral with regard to jurisdictional claims in published maps and institutional affiliations. 